\title{
General Intelligence: An Analysis of the Saudi Students' General Mental Ability and Elementary Cognitive Task Performance
}

\author{
Hesham Hanafy Alasali ${ }^{1}$ \\ ${ }^{1}$ King Saud University, Saudi Arabia \\ Correspondence: Hesham Hanafy Alasali, King Saud University, Saudi Arabia.
}

Received: August 30, 2021

Accepted: November 1, 2021

Online Published: November 24, 2021

doi:10.5539/ijps.v13n4p75

URL: https://doi.org/10.5539/ijps.v13n4p75

\begin{abstract}
Previous studies on human intelligence has revealed that varied factors influence cognitive performance, and some studies have hypothesized the presence of "general intelligence" ( $\mathrm{g}$ factor) that is responsible for intelligence. However, most studies have been centered on Western cultures. This study thus examines the Saudi students and the $\mathrm{g}$ factor hypothesis. A group of Saudi college students participated in a battery of general mental ability tests and elementary cognitive tasks and were assessed for the influence of various factors on cognitive performance. The study results revealed an increase in the average of Saudi students' intelligence compared to the results of the previous studies, but it did not yield a $\mathrm{g}$ factor.
\end{abstract}

Keywords: intelligence, general factor, fluid intelligence, elementary cognitive tasks, mental speed

\section{Introduction}

Human abilities span over enormous range; physical strength, intelligence, musical talent, and many more characteristics differ from person to person. Spearman (1904) suggested that there may be general intelligence (g factor) underlying the performance of cognitive tasks. This $\mathrm{g}$ factor can explain individual differences in general mental ability, and he described this factor as "mental energy." Mental speed approach developed this hypothesis and attributed the differences in the $\mathrm{g}$ factor to biological determinants, particularly the properties of the brain (e.g., Eysenck, 1986; Vernon, 1987; Jensen, 1998). According to this approach, the biological determinants are neural efficiency, nerve conduction velocity, and the speed of performing elementary cognitive tasks. Jensen $(1998,2000)$ suggests that half of the performance variance on intelligence quotient (IQ) tests is due to a variation in the $\mathrm{g}$ factor.

Several studies conducted to review intelligence have procured results that confirm the $\mathrm{g}$ factor hypothesis; these results were particularly prevalent in studies related to relationships between intelligence and information processing speed (Deary, Der, \& Ford, 2001; Nettelbeck, Edwards, \& Vreugdenhil, 1986; Vernon, 1983; Schubert, Hagemann, \& Frischkorn, 2017; Injoque-Ricle, Barreyro, Formoso, \& Burin, 2018; Frischkorn, Schubert, \& Hagemann, 2019). Additionally, the results indicate differences in $\mathrm{g}$ factor between men and women (Jorm, Anstey, Christensen, \& Rodgers, 2004; Jackson \& Rushton, 2006) and participants of different races (Jensen, 1969; Lynn, 2006; Rushton \& Jensen, 2010). Moreover, linkages prevail between the g factor and some neuroanatomical determinants, such as the size of the skull, the total brain volume (Rijsdijk, Vernon, \& Boomsma, 1998; Rushton \& Ankney, 2009), brain structure, brain function (Jung \& Haier, 2007; van der Linden, Dunkel, \& Madison, 2017; Bajaj et al., 2018), and genetics (Deary, Johnson, \& Houlihan, 2009; Lee, McGue, Iacono, Michael, \& Chabris, 2019). A relationship also existed between the $\mathrm{g}$ factor differences and life outcomes such as job performance (Schmidt \& Hunter, 1998), longevity (Gottfredson \& Deary, 2004), academic achievement (Jensen, 1993), career, and social life (Gottfredson, 1997, 2004; Kane \& Brand, 2003). Similarly, the results revealed a relationship between the performance of cognitive abilities tests and societal phenomena such as economic growth, average income (Lynn \& Vanhanen, 2006), and scores on international tests in science and mathematics (Rindermann, 2007; Kaufman, Reynolds, Liu, Kaufman, \& McGrew, 2012). These findings have led some researchers to classify human societies in the light of their intelligence scores (e.g., Lynn, 2006; Lynn \& Vanhanen, 2006).

Unfortunately, much of the evidence supporting the $\mathrm{g}$ factor came from nonrepresentative samples, most of which were centered on the Western culture (Arnett, 2008). Moreover, cultural factors were not considered when preparing and applying IQ tests. There are also doubts about whether the studies met the measurement 
equivalence (e.g., Sternberg, 2000; Sternberg, Grigorenko, \& Kidd, 2005; Nisbett, 2009; Wicherts, 2018). Nevertheless, the assertion that differences in the performance of the IQ tests are only due to cognitive factors is questionable (Richardson, 2002).

Accumulated empirical evidence falsifies the logic of classifying societies. One of the most important evidence is the Flynn effect (Flynn, 1984) that exhibits increase in the levels of raw intelligence from generation to generation. This increase cannot be attributed to genetic mutations; however, it can be attributed to economic growth, development of educational programs, social development, and health care (Flynn, 2007). Results documenting an increase in the IQ over time have been published in a number of developing countries, including Kenya (Daley, Whaley, Sigman, Espinosa, \& Neuman, 2003), the Dominican Republic (Meisenberg, Lawless, Lambert, \& Newton, 2006), Brazil (Colom, Flores-Mendoza, \& Abad, 2007), Sudan (Khaleefa, Sulman, \& Lynn, 2009), Saudi Arabia (Batterjee, Khaleefa, Ali, \& Lynn, 2013), and Egypt (Abdel-Khalek \& Lynn, 2016; Essa, Abdelrasheed, Bakhiet, Cheng, Dwieb, \& Lynn, 2016).

Notably, the studies conducted in Arab societies aimed to measure only the level of intelligence and did not seek to examine the $\mathrm{g}$ factor hypothesis. Therefore, by using this foundation, the current study focuses on administrating a battery of elementary cognitive tasks and general mental abilities tests to Saudi students. The performance of some of these elementary cognitive tasks requires automated processes, while performing others tasks require controlled processes. Therefore, it is important to examine whether these tasks are connected to the factors according to the performance processes or the nature of the processes, i.e., automatic or controlled. For example, the Stroop effect was not calculated by subtracting the difference between the conditions of congruent and incongruent conditions. Rather, data for each condition were computed separately in the factor analysis.

\section{Method}

\subsection{Participant}

The study sample included 62 students from the Department of Psychology at King Saud University, Riyadh, Saudi Arabia. Their ages ranged between 18 and 22 years, with an average age of 20.7 years and a standard deviation of 1.33 . They all volunteered to participate in the study.

\subsection{Measures}

This study employed various measurement methods to assess students and create data for analysis. These methods are listed below.

Standard Progressive Matrices (SPM) (Raven, Court, \& Raven, 1994): This method was used to assess pure general intelligence (see, e.g., Jensen, 1998). It had been used in past research involving several Arab samples (e.g., Abdel-Khalek \& Lynn, 2016; Essa et al., 2016).

Stroop Color-Naming Task: This task, through a computerized version, measured attention control (Macleod, 1991). The task included two experimental conditions, namely, congruence between the name and the writing color and incongruence between the name and the writing color.

Eriksen Flanker Task (EFT): This task measured selective attention (Eriksen \& Eriksen, 1974). In this task, participants were asked to press the correct response key when the target stimulus appeared. The task included two experimental conditions, namely, congruence between the target stimulus and the flanked stimuli and incongruence between the target stimulus and the flanked stimuli.

English Letter-Matching Task (ELMT): This task, devised by Posner and Mitchell (1967), measured the speed of retrieval from the long-term memory. The participants were asked to identify whether two letters had the same name by pressing the correct response key. The task included two experimental conditions, namely, a congruent condition in which the letter pairs are physically identical and have the same name and an incongruence condition in which the letter pairs are physically different and have a different name.

Arabic Letter-Matching Task (ALMT): The participants were asked to identify whether two Arabic letters had the same name by pressing the correct response key. The task included two experimental conditions, namely, a congruent condition in which the letter pairs are physically identical and have the same name and an incongruent condition in which the letter pairs are physically different and have a different name.

Processing Efficiency Task (PET): This task mainly involved performing simple math operations such as subtraction or addition. When performing this task, the participants were engaged in 10 experimental attempts in which they were asked to judge the accuracy of the result of a math equation. The experimental attempt began with Screen A displaying a simple math operation (for example, $5+4=9$ ). The participants had to press a specific response key if the result was correct and press a different response key if the result was incorrect. The 
participants had to remember the result, irrespective of the accuracy. Then, Screen B appeared (for example, $6+$ $2=9$ ). Screen $B$ also required the verification of an equation and the temporary retention of the result. The final screen comprised an equation to be verified, displaying the results of Screens A and B (for example, A + B = 18). In this case, the load on the simple short-term memory capacity remained constant in all experimental attempts. The differences in performance reflected the efficiency of processing independently of the working memory capacity (Burgaleta \& Colom, 2008).

Working Memory Capacity Task (WMCT): This task involved measuring the ability of the participants to maintain the results of a varying number of math equations. Each experimental attempt began with a math equation (for example, $3+4=7$ ). The participants had to assert whether the answer was correct by pressing a specific response key and had to remember the result, regardless of its accuracy. The experimental attempts varied in the number of math equations (between 3 and 7 equations). After presenting the final equation, the subjects were asked to write an equation using the results of the experimental attempts.

Saudi's General Aptitude Test (SGA): The participants undertook this test during the last year of high school. This test constituted a number of subtests that measured practical and verbal abilities. The participants' scores were expressed on a scale of 100 points, and score data were obtained from them.

\section{Results}

Data were analyzed using the SPSS statistical analysis package. Initially, a Kaiser-Meyer-Olkin test, which measures the sampling adequacy of each variable, was performed, and its value was 0.70 . Additionally, Bartlett's test of sphericity was performed, and its value was $p<0.000$. This aspect indicated that the correlation matrix was not 0 . These results demonstrated that the sample data were suitable for performing exploratory factor analysis. The principal component analysis was employed with Varimax rotation of factors and eigenvalues $>1$.

In all, four factors were extracted, and they explained $70 \%$ of the total variance. The first factor explained $23 \%$ of the total variance; the saturation of tasks of this factor ranged from 0.59 to 0.79 . The second factor explained $19 \%$ of the total variance, and the saturation of tasks of this factor ranged from 0.91 to 0.92 . The third factor explained $14 \%$ of the total variance; the saturation of tasks of this factor ranged from 0.901 to 0.904 . The fourth factor explained $12 \%$ of the total variance, and the saturation of tasks of this factor ranged from 0.45 to 0.87 . Table 2 presents the results of the exploratory factor analysis.

Table 1. Descriptive statistics

\begin{tabular}{llll}
\hline Task & $\mathrm{N}$ & Mean & Std. Deviation \\
\hline Arabic Letter-Matching Task & & & \\
Congruent (CALMT) & 62 & 680.7468 & 132.87872 \\
Incongruent (IALMT) & 62 & 791.5452 & 200.43522 \\
English Letter-Matching Task & & & \\
Congruent (CELMT) & 62 & 806.0885 & 187.25840 \\
Incongruent (IEALMT) & 62 & 1191.3037 & 318.37154 \\
Stroop Color-Naming Task & & & \\
Congruent (CSCNT) & 62 & 745.2296 & 318.01711 \\
Incongruent (ISCNT) & 62 & 758.8209 & 338.97432 \\
Eriksen Flanker Task & & & \\
Congruent (CEFT) & 62 & 552.8421 & 164.82103 \\
Incongruent (IEFT) & 62 & 619.8931 & 227.12628 \\
Processing Efficiency Task & 62 & .8080 & .18765 \\
Working Memory Capacity Task & 62 & 3.1113 & 1.03751 \\
Standard Progressive Matrices & 62 & 103.5161 & 10.12826 \\
Saudi's General Aptitude Test & 62 & 71.9839 & 7.00700 \\
\hline
\end{tabular}


Table 2. Results of the exploratory factor analysis

\begin{tabular}{lllll}
\hline Tasks & \multicolumn{3}{l}{ Factors } & \\
\cline { 2 - 5 } & 1 & 2 & 3 & 4 \\
\hline IEALMT & .786 & & & \\
CALMT & .756 & & & \\
CELMT & .743 & & & \\
Working Memory Capacity Task & -.655 & & & \\
IALMT & .589 & & & \\
CSCNT & & .917 & & \\
ISCNT & & .911 & & \\
CEFT & & & .904 & \\
IEFT & & & .901 & \\
Saudi's General Aptitude Test & & & & .868 \\
Standard Progressive Matrices & & & & .601 \\
Processing Efficiency Task & & & & .457 \\
Variance Explained (\%) & 23.036 & 19.512 & 14.512 & 11.843 \\
Total Variance Explained (\%) & & & & 68.903 \\
\hline
\end{tabular}

\section{Discussion}

The study results reveal a higher average IQ for Saudi students compared to the results of the students who participated in Lynn and Vanhanen (2002). Moreover, the rate of this increase is consistent with the effect reported in Flynn $(1984,2007)$; this study's rate of increase is consistent with the increase in average intelligence achieved by some Arab and developing countries during the past decades (see, e.g., Daley et al., 2003; Meisenberg et al., 2006; Colom et al., 2007; Khaleefa et al., 2009; Batterjee et al., 2013; Abdel-Khalek \& Lynn, 2016). This increase can be attributed to the increase in income and social development programs, improved health care, decreased illiteracy, and technological developments.

The results of this study did not reveal a $\mathrm{g}$ factor. On the contrary, the general mental ability tests' results were influenced by four factors; surprisingly, even the elementary cognitive tasks' results were influenced by various factors. However, these results are not consistent with the g factor hypothesis (see, e.g., Carroll, 1993; Jensen, 1998) and instead suggest that the variables underlying individual differences in intelligence and other cognitive tasks cannot be attributed simply to the speed of processing alone or only to cognitive factors (see, e.g., Richardson, 2002; Kahneman, 2011). Thus, noncognitive factors may influence a participant's performance even when performing elementary cognitive tasks.

Working memory capacity negatively correlated with the first factor. This aspect can be explained in light of the fact that the speed of information processing reduces the load on the limited working memory capacity and saves the limited information processing system resources to perform other mental processes. Additionally, the attention component of working memory requires faster information processing (Fry \& Hale, 1996; Jensen, 1998; Kail \& Salthouse, 1994; Salthouse, 1996).

It was also observed that the Raven's Standard Progressive Matrices, which measures fluid intelligence, and Saudi's General Aptitude Test, which measures both fluid and crystalized intelligence, relied on one factor. This result can be explained in light of the Sternberg hypothesis (Sternberg, 2005), suggesting three types of intelligence, namely, creative, practical, and analytical. It appears that the performance in the Raven's Standard Progressive Matrices and Saudi's General Aptitude Test require analytical intelligence.

However, future research with large representative samples and numerous cognitive tasks is needed to extract the $\mathrm{g}$ factor.

\section{Acknowledgments}

"The author thanks the Deanship of Scientific Research and RSSU at King Saud University for their technical support". 


\section{References}

American Psychological Association. (1972). Ethical standards of psychologists. Washington, DC: American Psychological Association.

Anderson, C. A., Gentile, D. A., \& Buckley, K. E. (2007). Violent video game effects on children and adolescents: Theory, research and public policy. https://doi.org/10.1093/acprof:oso/9780195309836.001.0001

Beck, C. A. J., \& Sales, B. D. (2001). Family mediation: Facts, myths, and future prospects (pp. 100-102). Washington, DC: American Psychological Association. https://doi.org/10.1037/10401-000

Bernstein, T. M. (1965). The careful writer: A modern guide to English usage (2nd ed.). New York, NY: Atheneum.

Bjork, R. A. (1989). Retrieval inhibition as an adaptive mechanism in human memory. In H. L. Roediger III, \& F. I. M. Craik (Eds.), Varieties of memory \& consciousness (pp. 309-330). Hillsdale, NJ: Erlbaum.

Cress, C. M. (2009). Curricular strategies for student success and engaged learning [PowerPoint slides]. Retrieved from https://www.vtcampuscompact.org/2009/TCL_post/presenter_powerpoints/Christine\%20Cress\%20-020Cu rricular\%20Strategies.ppt

Driedger, S. D. (1998, April 20). After divorce. Maclean's, 111(16), 38-43.

Gibbs, J. T., \& Huang, L. N. (Eds.). (1991). Children of color: Psychological interventions with minority youth. San Francisco, CA: Jossey-Bass.

Gilbert, D. G., McClernon, J. F., Rabinovich, N. E., Sugai, C., Plath, L. C., Asgaard, G., ... Botros, N. (2004). Effects of quitting smoking on EEG activation and attention last for more than 31 days and are more severe with stress, dependence, DRD2 A 1 allele, and depressive traits. Nicotine and Tobacco Research, 6, 249-267. https://doi.org/10.1080/14622200410001676305

Goleman, D. (2009). What makes a leader? In D. Demers (Ed.), AHSC 230: Interpersonal communication and relationships (pp. 47-56). Montreal, Canada: Concordia University Bookstore. (Reprinted from Harvard Business Review, 76(6), 93-102, 1998).

Guignon, C. B. (1998). Existentialism. In E. Craig (Ed.), Routledge encyclopedia of philosophy (Vol. 3, pp. 493-502). London, England: Routledge.

Healey, D. (2005). Attention deficit/hyperactivity disorder and creativity: An investigation into their relationship (Unpublished doctoral dissertation). University of Canterbury, Christchurch, New Zealand.

Herculano-Houzel, S., Collins, C. E., Wong, P., Kaas, J. H., \& Lent, R. (2008). The basic nonuniformity of the cerebral cortex. Proceedings of the National Academy of Sciences, 105, 12593-12598. https://doi.org/10.1073/pnas.0805417105

Klimoski, R., \& Palmer, S. (1993). The ADA and the hiring process in organizations. Consulting Psychology Journal: Practice and Research, 45(2), 10-36. https://doi.org/10.1037/1061-4087.45.2.10

Kubrick, S. (Director). (1980). The Shining [Motion picture]. United States: Warner Brothers.

Liu, S. (2005, May). Defending against business crises with the help of intelligent agent based early warning solutions. Paper presented at the Seventh International Conference on Enterprise Information Systems, Miami, FL. Retrieved from https://www.iceis.org/iceis2005/abstracts_2005.htm

MacIntyre, L. (Reporter). (2002, January 23). Scandal of the Century [Television series episode]. In H. Cashore (Producer), The fifth estate. Toronto, Canada: Canadian Broadcasting Corporation.

McLuhan, M. (1970a). Culture is our business. New York, NY: McGraw-Hill.

McLuhan, M. (1970b). From cliche to archetype. New York, NY: Viking Press.

Mellers, B. A. (2000). Choice and the relative pleasure of consequences. Psychological Bulletin, 126, 910-924. https://doi.org/10.1037/0033-2909.126.6.910

Postman, N. (1979). Teaching as a conserving activity. New York, NY: Delacorte Press.

Postman, N. (1985). Amusing ourselves to death: Public discourse in the age of show business. New York, NY: Viking. 
Semenak, S. (1995, December 28). Feeling right at home: Government residence eschews traditional rules. Montreal Gazette, p. A4.

Strong, E. K. Jr., \& Uhrbrock, R. S. (1923). Bibliography on job analysis. In L. Outhwaite (Series Ed.), Personnel Research Series: Vol. 1. Job analysis and the curriculum (pp. 140-146). https://doi.org/10.1037/10762-000

\section{Copyrights}

Copyright for this article is retained by the author(s), with first publication rights granted to the journal.

This is an open-access article distributed under the terms and conditions of the Creative Commons Attribution license (http://creativecommons.org/licenses/by/4.0/). 